\title{
Intoxicaciones por bupropión: presentación de caso
}

\author{
David Mejía Rodríguez* \\ Laura Elisa Montoya Gonzales** \\ Marie Claire Berrouet Mejia***
}

\begin{abstract}
* Médico psiquiatra, magíster en bioética. Docente de psiquiatría de pregrado y posgrado de la universidad CES. Medellín. Antioquia. Colombia. **Médica psiquiatra. Docente de psiquiatría de pregrado y posgrado de la universidad CES. Psiquiatra Hospital General de Medellín. Antioquia. Colombia.

***Médico psiquiatra. Especialista en toxicología clínica. Magíster en epidemiología. Docente Universidad CES. Toxicóloga Hospital General de Medellín. Antioquia. Colombia.

Correspondencia: Dr. David Mejía Rodríguez. Médico psiquiatra, magíster en bioética. Docente de psiquiatría de pregrado y posgrado de la universidad CES. Medellín. Antioquia. Colombia. Correo electrónico: davidmejiapsiq@gmail.com
\end{abstract}

\section{Resumen}

En Colombia las intoxicaciones autoinfligidas vienen en aumento en los últimos años, la principal causa son los medicamentos y dentro de éstos, los psicofármacos. El bupropión es un antidepresivo, inhibidor débil de la recaptación de dopamina y noradrenalina; está aprobado para el tratamiento del trastorno depresivo mayor y se ha utilizado en otras indicaciones como: depresión bipolar, abandono del cigarrillo, trastorno por déficit de atención e hiperactividad (TDAH), obesidad y disfunción sexual. Sus principales efectos adversos son cardiovasculares y neurológicos. Esta serie de casos busca evidenciar las complicaciones asociadas a su toxicidad y resaltar algunos aspectos en el manejo de estas intoxicaciones; tales como el uso de la irrigación gastrointestinal y el uso de emulsiones lipídicas. MÉD. UIS. 2020;33(2):117-121.

Palabras clave: Toxicidad. Psiquiatría. Bupropión. Convulsiones.

\section{Bupropion toxicity: a risk to consider}

\begin{abstract}
In Colombia, self-inflicted drug poisonings have been increasing in recent years, and within these, psychotropic drugs. The vast majority of psychoactive drug poisonings are explained by SSRI tricyclic antidepressants and anticonvulsants. Bupropion is an antidepressant, dopamine and norepinephrine reuptake inhibitor; approved for the treatment of major depressive disorder and used in other indications such as: bipolar depression, smoking cessation, attention deficit disorder the importance of this series of cases is to sensitize health personnel about the increase in prescription and therefore more Toxicity risks associated with this medicine. There is little literature on the management of bupropion toxicity, understanding these and it is important to highlight its amphetamine-like structure that explains neurological risks such as seizures and cardiovascular events as arrhythmias, although it is true that there is no consensus on the management, it is worth highlighting the usefulness of lipid emulsions This series of cases, being the first in Colombia, seeks to highlight the complications associated with its toxicity and highlight some aspects in the management of this poisoning; such as the use of gastrointestinal irrigation and the use of lipid emulsions. MÉD.UIS. 2020;33(2):117-121.
\end{abstract}

Keywords: Toxicity. Bupropion. Seizures. Psiquiatry.

¿Cómo citar este artículo?: Mejía D, Montoya LE, Berrouet MC. Intoxicaciones por bupropión: presentación de caso. MED.UIS. 2020;33(2):117-121. doi: 10.18273/revmed.v33n2-2020014 


\section{Introducción}

El bupropión es un antidepresivo aprobado por la Food and Drug Administration (FDA) para el tratamiento del trastorno depresivo mayor y el trastorno afectivo estacional'. En el 2007 en los Estados Unidos el bupropión fue el cuarto antidepresivo más prescrito. Un estudio realizado por Sherida se demostró que el bupropion era el segundo antidepresivo con más problemas de toxicidad luego de iniciada la terapia².

El bupropión ha aumentado en los últimos 4 años en los intentos suicidas de los adolescentes, además al compararlo con la ingesta de antidepresivos tricíclicos, las ingestas de bupropión fueron más severas y con mayor riesgo de ser ingresadas a $\mathrm{UCl}{ }^{3}$. Un estudio en Finlandia que revisó casos postmortem durante período de 5 años (20092013) donde evaluaron los hallazgos toxicológicos encontraron 30 casos en los que se determinó que la intoxicación era la causa de la muerte, de estos, 25 (83\%) fueron suicidios ${ }^{4}$. En Colombia según el Instituto de Vigilancia de Medicamentos y Alimentos (INVIMA) está aprobado para episodios depresivos mayores ${ }^{5}$. Como usos no aprobados es utilizado en: depresión bipolar, abandono del consumo de cigarrillo, trastorno por déficit de atención e hiperactividad (TDAH), obesidad y disfunción sexual, en Colombia se puede conseguir sin formula medica, no hay reportes de esta intoxicación ${ }^{1,5}$.

Recientemente Overberg et al. realizaron un estudio descriptivo de los datos reportados desde junio de 2013 hasta diciembre de 2017 para exposiciones de adolescentes (edades 10-19) a ISRS o bupropion, de los 30026 casos durantes el estudio, los ISRS representaron casi el 60\%, mientras que el bupropión se informó en el $11,7 \%$. La exposición al bupropión se asoció significativamente con la muerte (0.23\% vs $0 \% ; p<.001)$ o un resultado grave $(58.1 \%$ vs 19\%; $p<.001)$, así como los 10 efectos clínicos más comunes, incluidas las convulsiones (27.0\% vs $8.5 \%$; $p<.001)$ y alucinaciones ( $28.6 \%$ vs $4.3 \%$; $p$ <.001). La exposición al bupropión se asoció significativamente con la necesidad de reanimación cardiopulmonar ( $0.51 \%$ vs $0.01 \%$; $p<.001$ ), intubación ( $4.9 \%$ vs $0.3 \%$; $p<.001)$, vasopresores ( $1.1 \%$ vs $0.2 \% ; p<.001)$ y uso benzodiacepinas (34.2\% vs 5.5\%; $p<.001)^{6}$.

Su mecanismo de acción es la inhibición débil de la recaptación de dopamina y noradrenalina, si bien es cierto guarda una relación con la serotonina, de forma terapéutica no inhibe la recaptación de serotonina, pero en sobredosis puede presentar hallazgos de un síndrome serotoninergico ${ }^{7,8}$. Dentro de sus efectos adversos, se destacan efectos cardiovasculares, como: taquicardia, dolor torácico e hipertensión y dentro de los efectos neurológicos: insomnio, cefalea, agitación, mareo, acatisia, distonía y temblor. En sobredosis se asocia a efectos tóxicos neurológicos y cardiovasculares ${ }^{6,7,8}$

El objetivo de estos casos es que el lector reconozca que su toxicidad se presenta frecuentemente con signos y síntomas neurológicos, siendo común las convulsiones y alteraciones cardiovasculares, adicionalmente, se busca que se identifiquen las emulsiones lipídicas dentro del tratamiento.

\section{Presentación de los casos}

\section{Caso clínico número uno}

Paciente femenina de 18 años, con antecedentes de trastorno depresivo mayor y trastorno por uso de sustancias (cocaína, marihuana y éxtasis), en manejo ambulatorio por psiquiatría con venlafaxina 75 mg día y bupropion 30omg día. Es llevada al hospital luego de ser encontrada por su familia convulsionando; ingresa como triage II en estado postictal, signos vitales: presión arterial 150/90, frecuencia cardiaca 100 latidos por minuto, frecuencia respiratoria 18 respiraciones por minuto, con una saturación de oxígeno de $92 \%$ al aire ambiente, el resto del examen físico en límites normales. Con exámenes complementarios e ingreso (ionograma, hemoleucograma y electrocardiograma) en límites normales se solicitan pruebas para tóxicos en orina que se reportan positivos para anfetaminas 2 horas después de su ingreso refiere que se tomado 6 comprimidos de bupropion con fines suicidas, se decide entonces dejarla en observación hasta que pasen cuatro vidas del medicamento, (la vida media del bupropion es de 24 horas), ocho horas después del ingreso, presenta un nuevo episodio convulsivo, que mejora con la administración de $10 \mathrm{mg}$ de diazepam IV. Continúa en observación por 60 horas, sin nuevas complicaciones neurológicas ni cardiovasculares y se remite a institución de salud mental para continuar manejo por psiquiatría debido su riesgo suicida.

\section{Caso clínico número dos}

Paciente femenina de 22 años, con antecedentes de trastorno depresivo mayor y trastorno de la 


\section{Mayo - agosto}

conducta alimentaria no especificado, en manejo con bupropion $300 \mathrm{mg}$ al día, venlafaxina 150 $\mathrm{mg}$ al día y trazodona $100 \mathrm{mg}$ al día durante el último mes; ingresa a una institución de mediana complejidad cuatro horas después de ingerir una cantidad desconocida de trazodona, clonazepam y bupropion. Se encuentra somnolienta, con presión arterial de 100/60, frecuencia cardiaca de 75 latidos por minuto, frecuencia respiratoria de 17 respiraciones por minuto, saturando $94 \% \sin$ oxígeno, el resto del examen físico dentro de límites normales. Inmediatamente se realiza manejo con lavado gástrico y carbón activado; posteriormente, se documenta episodio convulsivo tónico clónico generalizado que responde a $10 \mathrm{mg}$ de diazepam IV, recuperando el nivel de conciencia; 10 minutos después, presenta un segundo episodio de las mismas características, por lo tanto, se administran otros $10 \mathrm{mg}$ de diazepam IV y se hace impregnación con fenitoína $18 \mathrm{mg} / \mathrm{kg}$. Se inicia proceso de remisión a un servicio de alta complejidad, durante el traslado presenta ritmo de paro no desfibrilable, se inicia manejo con compresiones y adrenalina sin respuesta; fallece durante la remisión. No se tuvieron datos de si se realizó necropsia.

\section{Caso clínico número tres}

Paciente femenina de 40 años, con antecedente de trastorno afectivo bipolar, en manejo con bupropion $300 \mathrm{mg} /$ día desde hace una semana. Ingresa a un servicio de alta complejidad, como triage I, luego de cuatro episodios convulsivos tónico clónico generalizados tras ingerir 140 comprimidos de bupropion; a su llegada, ante el riesgo neurológico y ventilatorio se asegura la vía aérea y se traslada a la unidad de cuidados intensivos, donde ingresa sin repuesta a estímulos dolorosos; signos vitales: presión arterial 110/70, frecuencia cardiaca 100 latidos por minuto, saturación de oxígeno 96\%. Se continúan documentando movimientos anormales, se inicia infusión de midazolam a $5 \mathrm{mg} /$ hora y emulsiones lipídicas al $20 \%: 1.25 \mathrm{ml} / \mathrm{kg}$ en bolo, con posterior administración de $0.25 \mathrm{ml} / \mathrm{kg}$ para una hora. Un día después, requiere soporte vasopresor con norepinefrina a $0.02 \mathrm{mcg} / \mathrm{kg} / \mathrm{min} ; 24$ horas más tarde presenta fiebre, disautonomía y rigidez, se sospecha un síndrome neuroléptico maligno y se adiciona bromocriptina $2.5 \mathrm{mg} /$ día con poca mejoría; seis horas después presenta asistolia, se realizan maniobras de reanimación durante 25 minutos, sin respuesta y la paciente fallece.

\section{Discusión}

El bupropion es un antidepresivo utilizado en diferentes indicaciones: en el trastorno depresivo mayor, el bupropion ha sido estudiado en todas sus formulaciones: IR, SR y XL. El bupropion IR a dosis de $300 \mathrm{mg} /$ día es más eficaz al placebo ${ }^{7,8}$ y cuando se comparó con antidepresivos tricíclicos demostró una eficacia similar ${ }^{9,10,11}$.

Con respecto a los efectos adversos de este medicamento se han descrito dentro de los más frecuentes, en más de un 10\% taquicardia, insomnio, cefalea pérdida de peso, temblor, arritmias, hipotensión, distonia, acatisia mialgias y convulsiones ${ }^{12-14}$

Los casos reportados en esta serie están en concordancia por lo reportado por Overberg y colaboradores" ${ }^{11}$ donde se evidencia mayor frecuencia los síntomas neurológicos, cardiovasculares y la necesidad de reanimación. El primer caso clinico reportado va en consonancia con lo anteriormente descrtio; era una mujer que consumía solo psicofármacos. Otros autores como Dagan ${ }^{15}$ reportan casos similares al caso clínico reportado donde las convulsiones fueron posteriores a la ingesta.

Con respecto al riesgo cardiovascular, al ser la dopamina, una amina precursora de la noradrenalina y la adrenalina explica la hipertensión y taquicardia; en casos de toxicidad también se ha descrito shock cardiogénico luego de ingestas superiores a 12 gramos $^{16}$. Dentro de las manifestaciones electrocardiográficas más comunes están la prolongación del QRS y del QT, sin embargo la prolongación del QT no es tan frecuentemente descrita como con otros psicofármacos ${ }^{17,18}$. En cuanto al manejo de estos pacientes es importante tener en consideración la identificación temprana de este riesgo para la solicitud temprana y pertinente de ayudas diagnósticas como el electrocardiograma, sólo solicitado en uno de los $\operatorname{casos}^{17,18}$.

Con respecto al riesgo neurológico, desde la reintroducción del bupropion al mercado, ha tenido como precaución el riesgo de convulsiones, por lo tanto, se sugiere una dosis máxima de $450 \mathrm{mg} /$ día y se ha propuesto que en pacientes con riesgo de convulsiones no se utilice, entendiendo que con dosis tan bajas como $600 \mathrm{mg}$ se han reportado convulsiones ${ }^{18-21}$ 
No solo el principio activo explica la neurotoxicidad, también sus metabolitos activos como: eritrohidrobupropion y el treohidrobupropion, que adicionalmente tienen un anillo de anfetamina en su estructura, que explican las pruebas cruzadas positivas, como ocurrió en el primer caso $^{18-22}$

Las convulsiones asociadas al bupropion se explican a través de cuatro mecanismos: el imbalance entre neurotransmisores inhibitorios como el GABA (ácido gama animo butírico) y neurotransmisores excitatorios, con aumento de los segundos sobre los primeros ${ }^{18-22}$; alteraciones en los canales de sodio que favorecen procesos de despolarización; Aumento en la concentración de aminas biógenas como dopamina y noradrenalina antagonismo de receptores inhibitorios como los de glicina o GABA ${ }^{20-22}$

Las convulsiones en el paciente intoxicado son relativamente frecuentes (entre 8,4 - 28\%), la presentación más común son convulsiones tónico clónicas generalizadas y auto resolutivas, sin embargo, menos del $5 \%$ de los pacientes con convulsiones de etiología tóxica van a presentar un estado epiléptico ${ }^{11,13,15,21}$

La literatura disponible acerca de las convulsiones secundarias a la intoxicación por bupropion, reconoce la ausencia de antídotos específicos y plantea el aseguramiento de la vía aérea y la utilización de benzodiacepinas como primera línea de manejo; la fenitoína, que puede bloquear los canales de sodio, no sería el medicamento ideal en estos casos y se propone el uso de emulsiones lipídicas dado el perfil lipofílico del bupropion ${ }^{11,18-22}$. En los casos descritos, se evidenció el uso de benzodiacepinas, en un caso se usó fenitoína y en otro caso se usaron emulsiones lipídicas.

Las emulsiones lipídicas son compuestos formados por triglicéridos y fosfolípidos que se utilizan ampliamente como suplementos nutricionales y como vehículo de algunos medicamentos como el propofol, dentro de las principales teorías sobre su modo de acción se encuentran: la capacidad de atrapar y aislar del torrente sanguíneo a medicamentos liposolubles, efecto conocido como lipid sink; la optimización del metabolismo del miocardiocito; el hecho de que se constituyen en fuente de adenosin trifosfato (ATP) y adicionalmente, la modulación permitida por los lípidos de los potenciales de acción de los canales de sodio y de calcio de la membrana celular, activándolos en su presencia y ejerciendo un efecto cardioprotector de esta forma ${ }^{23-26}$

La toxicidad por bupropion ha sido uno de los escenarios para evaluar la utilidad de las emulsiones lipídicas, es importante resaltar que no son el tratamiento de primera línea ${ }^{23-26}$. En el estudio LIPAEMIC, se recolectó información entre 2009 y 2012 acerca del uso de emulsiones lipídicas en pacientes intoxicados, entre los desenlaces favorables se evidenció mejoría en la escala de coma de Glasgow y en las cifras de presión arterial27-31

En cuanto a las medidas de descontaminación iniciales, en casos de formas de liberación modificada, es importante la irrigación gastrointestinal total, la cual, busca hacer un barrido total del intestino al administrar polietilenglicol, que es una solución balanceada osmóticamente; en el paciente adulto se administra con una velocidad de $1500-2000 \mathrm{ml} /$ hora, buscando que las deposiciones se excreten claras y tiene utilidad en medicamentos de liberación extendida"1. Sin embargo, en los casos descritos las medidas de descontaminación no estaban indicadas, sólo se deben realizar en pacientes que estén alerta y una hora luego de la ingesta ${ }^{23}$.

\section{Conclusiones}

Aunque el bupropion es un antidepresivo eficaz, se deben tener en cuenta sus indicaciones, contraindicaciones y costoefectividad, es fundamental conocer los riesgos neurológicos y cardiovasculares asociados, para buscar siempre un balance entre seguridad y eficacia. En estos resultados al igual que en la literatura, se evidencia que a mayor dosis y coingestas se aumenta el riesgo de letalidad, de ahí radica la importancia de un reconocimiento temprano de los riesgos.

\section{Referencias bibliográficas}

1. Drug Safety and Availability [Internet]. U.S. Food \& Drug Administration. Silver Spring (MD): U.S. Food \& Drug Administration; 1985. Disponible en: http://www.accessdata.fda. gov/drugsatfda_docs/label/2016/021515s034lbl.pdf

2. Sheridan DC, Lin A, Horowitz BZ. Suicidal bupropion ingestions in adolescents: increased morbidity compared with other antidepressants. Clin Toxicol (Phila). 2018;56(5):360-4.

3. Sheridan DC, Lin A, Horowitz BZ. Suicidal bupropion ingestions in adolescents: increased morbidity compared with other antidepressants. Clin Toxicol (Phila). 2018;56(5):360-4.

4. Kriikku, P., \& Ojanperä, I. The relationship between bupropion and suicide in post-mortem investigations. Forensic science 
international. 2016;266:343-8.

5. Invima. Farmaco vigilancia [Internet]. Disponible en: http:// farmacovigilancia.invima.gov.co:8082/Consultas/consultas/ consreg encabcum.jsp

6. European Space Agency. ESA: Missions, Earth Observation: ENVISAT [Internet]. [Consultado 3 Jul 2012]. Disponible en: http://envisat.esa.int/

7. Overberg A, Morton S, Wagner E, Froberg B. Toxicity of Bupropion Overdose Compared With Selective Serotonin Reuptake Inhibitors. Pediatrics. 2019;144(2):e20183295.

8. Khan SR, Berendt RT, Ellison CD, Ciavarella AB, Asafu-Adjaye E, Khan MA, et al. Bupropion Hydrochloride. Profiles Drug Subst Excip Relat Methodol. 2016;41:1-30.

9. Sidlak AM, Koivisto KO, Marino RT, Abesamis MG. Serotonin toxicity from isolated bupropion overdoses [published online ahead of print, 2020 Apr 14]. Clin Toxicol (Phila). 2020;1-3.

10. Lineberry CG, Johnston JA, Raymond RN, Samara B, Feighner JP, Harto NE, et al. A fixed-dose (300 mg) efficacy study of bupropion and placebo in depressed outpatients. J Clin Psychiatry. 1990;51(5):194-9.

11. Feighner J, Hendrickson G, Miller L, Stern W. Double-blind comparison of doxepin versus bupropion in outpatients with a major depressive disorder. J Clin Psychopharmacol. 1986;6(1):27-32

12. Overberg A, Morton S, Wagner E, Froberg B. Toxicity of Bupropion Overdose Compared With Selective Serotonin Reuptake Inhibitors. Pediatrics. 2019;144(2): 1-13.

13. 13. Aplenzin (bupropion hydrobromide) [prescribing information]. Bridgewater, NJ: Bausch Health US, LLC; May 2020. Disponible en: https:/www.bauschhealth.com/Portals/25/ Pdf/PI/Aplenzin-PI.pdf

14. Chaudron LH, Schoenecker CJ. Bupropion and Breastfeeding: A Case of a Possible Infant Seizure. J Clin Psychiatry. 2004; 65(6):881-2.

15. Fava M. Prospective studies of adverse events related to antidepressant discontinuation. J Clin Psychiatry. 2006;67(4):14-21.

16. Dagan Y, Yager J. Severe bupropion XR abuse in a patient with long-standing bulimia nervosa and complex PTSD. Int J Eat Disord. 2018;51(10):1207-1209.

17. Givens, M. L., \& Gabrysch, J. (2007). Cardiotoxicity associated with accidental bupropion ingestion in a child. Pediatric emergency care, 23(4), 234-237.

18. Al-Abri, S. A., Orengo, J. P., Hayashi, S., Thoren, K. L., Benowitz, N. L., Olson, K. R. Delayed bupropion cardiotoxicity associated with elevated serum concentrations of bupropion but not hydroxybupropion. Clinical Toxicology. 2013; 51(10), 1230-4.

19. Morazin, F., Lumbroso, A., Harry, P., Blaise, M., Turcant, A., Montravers, P., et al. Cardiogenic shock and status epilepticus after massive bupropion overdose. Clin Toxicol. 2007. 45(7), 794-7.

20. Judge BS, Rentmeester LL. Antidepressant overdose-induced seizures. Psychiatr Clin North Am. 2013; 36(2):245-60.

21. Rivas-Coppola, M. S., Patterson, A. L., Morgan, R., Wheless, J. W. Bupropion overdose presenting as status epilepticus in an infant. Pediatric neurology. 2015;53(3): 257-261.

22. Starr, P., Klein-Schwartz, W., Spiller, H., Kern, P., Ekleberry, S. E., Kunkel, S. Incidence and onset of delayed seizures after overdoses of extended-release bupropion. Am J Emerg Med. 2009;27(8):911-915.

23. Noda AH, Schu U, Maier T, Knake S, Rosenow F. Epileptic seizures, coma and EEG burst-suppression from suicidal bupropion intoxication. Epileptic Disord. 2017;19(1):109-113.

24. Cárdenas-Álvarez JE, Berrouet-Mejía MC. Uso de las emulsiones lipídicas en intoxicaciones por anestésicos locales y otras intoxicaciones: evidencia actual. Rev CES Med 2014; 28(1): 77-90

25. Cave G, Harvey MG. Should we consider the infusion of lipid emulsion in the resuscitation of poisoned patients?. Crit Care. 2014;18(5):457.

26. Hoegberg LCG, Gosselin S. Lipid resuscitation in acute poisoning: after a decade of publications, what have we really learned?. Curr Opin Anaesthesiol. 2017;30(4):474-476.

27. Sirianni, A. J., Osterhoudt, K. C., Calello, D. P., Muller, A. A., Waterhouse, M. R., Goodkin, M. B., et al. Use of lipid emulsion in the resuscitation of a patient with prolonged cardiovascular collapse after overdose of bupropion and lamotrigine. Ann Emerg Med. 2008;51(4):412-5.

28. Rothschild, L., Bern, S., Oswald, S., Weinberg, G. Intravenous lipid emulsion in clinical toxicology. Scand J Trauma Resusc Emerg Med. 2010;18(1):51.

29. Levine, M., Hoffman, R. S., Lavergne, V., Stork, C. M., Graudins, A., Chuang, R., et al. Systematic review of the effect of intravenous lipid emulsion therapy for non-local anesthetics toxicity. Clin Toxicol. 2016;54(3):194-221.

30. Hoegberg LCG, Gosselin S. Lipid resuscitation in acute poisoning: after a decade of publications, what have we really learned?. Curr Opin Anaesthesiol. 2017;30(4):474-476.

31. Cave, G., Harvey, M., Willers, J., Uncles, D., Meek, T., Picard, J., et al. LIPAEMIC report: results of clinical use of intravenous lipid emulsion in drug toxicity reported to an online lipid registry. J Med Toxicol. 2014;10(2):133-142. 